\title{
A NOTE ON LOCALLY SOLUBLE, NORMAL CLOSURES OF CYCLIC SUBGROUPS
}

\author{
by HOWARD SMITH
}

(Received 15 June, 1992)

This brief note has the threefold purpose of improving on an earlier theorem of the author [4], gathering together some results on normal closures (with rank restrictions) which are more or less implicit in the literature and providing a few examples which indicate the impossibility of improving these results in one way or another. The proofs are mostly routine and usually omitted. Most of the relevant background material can be found in [3], and references to these results will often indicate that minor additional details (an easy induction, for example) are required. Throughout, $\langle x\rangle^{G}$ will denote the normal closure of the subgroup $\langle x\rangle$ of the group $G$. The usual notation is used for upper central and derived series.

If $G$ is locally nilpotent and $\langle x\rangle^{G}$ is finitely generated, then $\langle x\rangle^{G}$ is contained in $Z_{\imath}(G)$ for some finite $t$ (e.g. by Lemma 6.37 of [3]) and so, if $\langle x\rangle^{G}$ is finitely generated for all $x$, then $G$ is hypercentral of length at most $\omega$ and is of course a Fitting group (though not necessarily nilpotent). The following result, therefore, completes the description of the hierarchy of the "main classes" of locally nilpotent groups with all normal closures finitely generated (cf. [5]).

THEOREM 1. A group $G$ having all subgroups subnormal and $\langle x\rangle^{G}$ finitely generated for all $x$ is nilpotent.

Proof. Since each $\langle x\rangle^{G}$ has a descending $G$-invariant series of subgroups of finite index whose intersection is trivial, it follows that $G / Z(G)$ is residually finite. Theorem 1 of [6] gives the result.

In general, a group $G$ with all normal closures finitely generated is not locally max (where "max" denotes the property of satisfying the maximal condition for subgroups). This can be seen by considering $\operatorname{SL}(n, \mathbb{Z}), n \geq 3$, which has all of its noncentral normal subgroups finitely generated [3, Vol. 1, p. 173], or any finitely generated simple group without the maximal condition. However, denoting by $\mathfrak{X}$ the (relatively large) class of groups having an ascending normal series whose factors are locally (soluble or finite), we have the following.

THEOREM 2. If $G$ is locally $\mathfrak{X}$ and $\langle x\rangle^{G}$ is finitely generated for all $x$, then $\langle x\rangle^{G}$ satisfies max for all $x$, and so each $\langle x\rangle^{G}$ is polycyclic-by-finite and $G / Z(G)$ is residually finite.

Proof. With $G$ as given, let $H$ be a finitely generated normal subgroup of $G$ and suppose $H$ has an ascending $G$-invariant series of length $\alpha>0$. Then $\alpha$ is not a limit ordinal and so $H$ has a $G$-invariant subgroup $K$ having a series of length $\alpha-1$ with $H / K$ finite or soluble. If $H / K$ is finite, then $K$ is finitely generated and, by induction on $\alpha, K$ may be assumed to satisfy max. So $H$ has max. If $H / K$ is soluble then, for some $n, H^{(n)}$ lies in $K$ and is finitely generated as a $G$-group and hence finitely generated. Again by induction, $H^{(n)}$ has max. Factoring, we may suppose $H$ soluble. An easy induction on the derived length concludes the proof. 
Turning now to the property of finite (Prüfer) rank, we note that if $G$ is a locally nilpotent group having $\langle x\rangle^{G}$ of finite rank for all $x$ then $G$ is hypercentral [3, Vol. 2, p. 39], though not necessarily of length at most $\omega$, as is shown by the wreath product of a group of type $C_{p^{\infty}}$ by a cyclic group of order $p$, in which the normal closure of the "top group" is not nilpotent. In fact, Theorem $6(\mathrm{a})$ indicates that $\langle x\rangle^{G}$ need not even be soluble. However, if $G$ is torsion-free then (on account of $[3,6.37]$ ) the hypercentral length is at most $\omega$. With subnormality present we can say considerably more.

TheOREM 3. Let $H$ be a subnormal subgroup of defect at most $m$ in the group $G$. Suppose that $H$ is nilpotent of class at most $c$ and that $H^{G}$ has finite rank $r$. Then $H^{G}$ is nilpotent of class at most $f=f(m, c, r)$.

This improves on the theorem in [4], where it was required that $G$ be locally residually finite and that $H$ be cyclic.

COROLlaRY 4. If $G$ is generated by subnormal subgroups $\left\langle x_{\lambda}\right\rangle$ of defect at most $m$ such that $\left\langle x_{\lambda}\right\rangle^{G}$ has rank at most $r$, for each $\lambda$, then $G$ is nilpotent of bounded class.

These remarks on locally nilpotent groups with all normal closures of finite rank enable one to determine the various inclusions among such classes of groups as in [5].

Proof of Theorem 3. Let $F=\left\langle h_{1}^{g_{1}}, \ldots, h_{t}^{g_{\prime}}\right\rangle$, for some elements $h_{i}$ of $H, g_{i}$ of $G$. Each $\left\langle h_{i}^{g_{i}}\right\rangle$ has defect at most $m+c-1$ in $F$ and so each (finite) $p$-image of $F$ is generated by at most $r$ cyclic subgroups of bounded defect and hence has bounded nilpotency class. The result follows.

The corollary is a consequence of the following lemma.

LEMMA 5. Let $G$ be a group which is generated by subnormal subgroups $\left\langle x_{\lambda}\right\rangle$, each of defect at most $m$ in $G$, and let $A$ be a normal abelian subgroup of rank $r$ in $G$. Then $A \leq Z_{n}(G)$, where $n=n(m, r)$.

Proof. We may suppose $G$ is finitely generated and hence nilpotent. Denote by $T$ the torsion subgroup of $A$. Then $A / T \leq Z_{r}(G / T)$ and we quickly reduce to the case where $A$ is a finite $p$-group.

Then $\bar{G}=G / C_{G}(A)$ has rank at most $s=\frac{1}{2} r(5 r-1)$ (by $\left.[3,7.44]\right)$ and is generated by subgroups $\overline{\left\langle x_{\lambda}\right\rangle}$ of defect at most $m$, and hence (since $\bar{G}$ is a finite $p$-group) by at most $s$ such subgroups. By Theorem A of [2], $\bar{G}$ has bounded defect in the natural split extension $A] \bar{G}$. The result follows.

We note that if $G$ is a locally soluble group and $\langle x\rangle^{G}$ has finite rank for all $x$ then $G$ is hyperabelian $[3,10.39]$ but not necessarily of "length" at most $\omega$ (see Theorem 6(a) and (b)). Suppose in addition that $G$ is torsion-free. If, for each $r$, the product of all normal subgroups $H_{\lambda}$ of rank at most $r$ were soluble, it would follow that $G$ has hyperabelian length at most $\omega$. But this is indeed the case: firstly we note that each such $H_{\lambda}$ has a characteristic abelian series of bounded length whose factors are torsion-free or finite [3, 10.39 and 9.34]. The result then follows via successive applications of [3, 6.37 and 7.45]. (The above remarks parallel the earlier ones on locally nilpotent groups.)

Finally, we describe some examples.

THEOREM 6. There exist groups $G_{1}, G_{2}, G_{3}$ containing elements $x_{1}, x_{2}, x_{3}$ respectively such that 
(a) $G_{1}$ is locally nilpotent of finite rank but $\left\langle x_{1}\right\rangle^{G_{1}}$ is not soluble.

(b) $G_{2}$ is a locally soluble, torsion group of finite rank but $\left\langle x_{2}\right\rangle^{G_{2}}$ is not soluble.

(c) $G_{3}$ is a Baer group with finite abelian subgroup rank but $\left\langle x_{3}\right\rangle^{G_{3}}$ is not soluble.

Proof. A construction due to Kegel [3, Vol. 2, p. 179] provides, for each prime $p$, a finite $p$-group $H(p)$ of rank at most 9 such that the derived length of $H(p)$ tends to infinite with $p$. For each $p$, choose an element $a_{p}$ of $H(p)$ such that the derived length of the subgroups $\left\langle a_{p}\right\rangle^{H(p)}$ are unbounded.

(a) Write $H_{1}=\underset{p}{\operatorname{Dr}} H(p)$ and define $x_{1}$ in Aut $H_{1}$ via the assignment $x_{1}: h \rightarrow h^{a_{p}}$, $x_{1}^{-1}: h \rightarrow h^{a_{p}-1}$, for all $h \in H(p)$ and for each $p$. Let $\left.G_{1}=H_{1}\right]\left\langle x_{1}\right\rangle$. Then $\left\langle x_{1}\right\rangle^{G_{1}}$ contains $\left[H_{1},\left\langle x_{1}\right\rangle\right]$, which is not soluble, though $G_{1}$ is locally nilpotent and of finite rank.

(b) With $H_{1}$ as above, let $G_{2}$ denote the wreath product of $H_{1}$ by a cyclic group $\left\langle x_{2}\right\rangle$ of order 2. Then $H_{1}^{\left\langle x_{2}\right\rangle}=H_{1}\left[H_{1},\left\langle x_{2}\right\rangle\right]$, and so $\left[H_{1},\left\langle x_{2}\right\rangle\right]$ maps onto $H_{1}^{\left\langle x_{2}\right\rangle} \cong H_{1}$, which is not soluble.

(c) To establish (c) we need a construction due to Dark [1]. This time it is necessary to provide some more details - what the following amounts to is the choosing of a finite section of each of Dark's (infinite) $p$-groups, and then the introduction of an automorphism $x_{3}$ similar to $x_{1}$ above.

Let $p$ be a prime and let $U_{p}$ be the group of upper unitriangular matrices of degree $p+1$ over the field of $p$ elements. Then $U_{p}=\left\langle\epsilon_{i}: i=1, \ldots, p\right\rangle$, where, for each $i, \epsilon_{i}$ is the matrix with 1's on the main diagonal and in position $(i, i+1)$ and zeroes elsewhere. Also, $\left\langle\epsilon_{i}\right\rangle^{U_{p}}$ is abelian and $\epsilon_{i}^{p}=1$. Let $F$ be the free group on generators $c_{1}, \ldots, c_{p}$ and for each $\sigma \in \operatorname{Sym}\{1, \ldots, p\}=\Sigma_{p}$ define a homomorphism $\sigma^{*}: F \rightarrow U_{p}$ via the assignment $c_{i} \rightarrow \epsilon_{i \sigma}$. Let $K_{\sigma}=\operatorname{ker} \sigma^{*}$, and let $K$ be the intersection of all the $K_{\sigma}$ as $\sigma$ runs through $\Sigma_{p}$. So $H=F / K$ is a finite $p$-group of class $p$ and derived length $\left[\log _{2}(p+1)\right]+1$. For each $i$, let $y_{i}$ denote the image of $c_{i}$ under the natural homomorphism from $F$ onto $H$. Then $y_{i}^{p}=1$ and $\left\langle y_{i}\right\rangle^{H}$ is abelian. Let $\pi$ be the cycle $(1, \ldots, p) \in \Sigma_{p}$ and define $\theta \in$ Aut $F$ via $c_{i} \theta=c_{i \pi}$, for all $i$. If $g \in F$ and $\sigma \in \Sigma_{p}$ then $g \in K_{\sigma} \theta$ if and only if $g \in K \pi^{-1} \sigma$. So $\theta$ permutes the $K_{\sigma}$ and thus leaves $K$ invariant. Then $\pi$ induces an automorphism $\alpha$ of $H$ such that $y_{i} \alpha=y_{i \pi}$, and clearly $\alpha^{p}=1$ and so $\left.J=H\right]\langle\alpha\rangle$ is again a finite $p$-group. Now $\left\langle y_{1}\right\rangle^{J} \geq\left\langle y_{1}\right\rangle^{\langle\alpha\rangle}=$ $\left\langle y_{1}, \ldots, y_{p}\right\rangle=H$ and $\left\langle y_{1}\right\rangle \triangleleft\left\langle y_{1}\right\rangle^{H}$ (which is abelian) and so $\left\langle y_{1}\right\rangle \triangleleft^{3} J$. Since $\left\langle y_{1}\right\rangle^{J}$ has derived length $\left[\log _{2}(p+1)\right]+1$, we see that $\left[\left\langle y_{1}\right\rangle, J\right]$ has derived length at least $d_{p}=\left[\log _{2}(p+1)\right]$. Now effect the above construction for each prime $p$, denoting the resulting split extension by $J_{p}$ and the element corresponding to $y_{1}$ by $h_{p}$. Then $L_{p}=\left[J_{p},\left\langle h_{p}\right\rangle\right]$ has derived length at least $d_{p}$, and $\left\langle h_{p}\right\rangle\left\langle^{3} J_{p}\right.$. Let $J$ be the direct product of all the $J_{p}$ and define $x_{3}$ in Aut $J$ by $a^{x_{3}}=a^{h_{p}}, x^{x_{3}^{-1}}=a^{h_{p}^{-1}}$, for all $a \in J_{p}$, and for each prime $p$. Set $\left.G_{3}=J\right]\left\langle x_{3}\right\rangle$. Then $\left[G_{3},\left\langle x_{3}\right\rangle\right] \leq J$, and $\left[J_{.4}\left\langle x_{3}\right\rangle\right]=\operatorname{Dr}\left[J_{p, 4}\left\langle h_{p}\right\rangle\right]=1$. Thus $\left\langle x_{3}\right\rangle$ is subnormal in $G_{3}$, which is therefore a Baer group. Clearly the $p$-rank of $G_{3}$ is finite, for each prime $p$, as is the torsion-free rank. However, $\left\langle x_{3}\right\rangle^{G_{3}} \geq \operatorname{Dr} L_{p}$, which is not soluble.

\section{REFERENCES}

1. R. S. Dark, A prime Baer group. Math. Z. 105 (1968), 294-298.

2. J. C. Lennox and S. E. Stonehewer, The join of two subnormal subgroups. J. London Math. Soc. (2) 22 (1980), 460-466. 
3. D. J. S. Robinson, Finiteness conditions and generalized soluble groups (2 vols.). Springer, Berlin-Heidelberg-New York (1972).

4. H. Smith, A note on Baer groups of finite rank. Math. Z. 184 (1983), 139-140.

5. H. Smith, Some remarks on locally nilpotent groups of finite rank. Proc. Amer. Math. Soc. 92 (1984), 339-341.

6. H. Smith, Hypercentral groups with all subgroups subnormal II. Bull. London Math. Soc. 18 (1986), 343-348.

Department of Mathematics

BUCKNELL UNIVERSITY

LEWISBURG, PA 17837

U.S.A. 